\title{
A EDUCAÇÃO A DISTÂNCIA NOS CURSOS DE GRADUAÇÃO EM ENFERMAGEM: APLICAÇÃO E EFETIVIDADE
}

\author{
EDUCACIÓN A DISTANCIA EN LOS CURSOS DE GRADUACIÓN EN \\ ENFERMERÍA: APLICACIÓN Y EFECTIVIDAD
}

\section{DISTANCE EDUCATION IN NURSING GRADUATION COURSES: APPLICATION AND EFFECTIVENESS}

\author{
Erika ZAMBRANO TANAKA ${ }^{1}$ \\ Dulcegleika Villas Boas SARTORI ${ }^{2}$ \\ Larissa Ribeiro FERREIRA ${ }^{3}$ \\ Lucas Justiniano BERMEJO ${ }^{4}$
}

RESUMO: A educação a distância $(\mathrm{EaD})$ foi reconhecida pela primeira vez na Lei de Diretrizes e Bases da Educação (LBD) em 1996, e refere-se a uma modalidade de ensino-aprendizagem onde os alunos e tutores estão separados, contudo conectados por meio de diversas tecnologias como a internet. A EaD vem crescendo gradativamente com o passar dos anos, e tem sido constante a sua utilização em diversas modalidades de cursos, sejam técnicos, de graduação ou pós-graduação. Os cursos de enfermagem, assim como outros cursos da área da saúde, têm usado a EaD com o intuito de facilitar o acesso a aprendizagem, oferecendo ao aluno ensino de qualidade e conhecimento de novas tecnologias. Este estudo foi desenvolvido com o intuito de analisar a $\mathrm{EaD}$, assim como sua aplicação nos cursos de enfermagem. Para tanto foi realizada uma revisão de literatura qualitativa de pesquisa teórica. Foram retirados artigos em bases de dados nacionais publicados no período de 2002 a 2014.

PALAVRAS-CHAVE: Educação a distância. Enfermagem. Aplicabilidade na enfermagem.

RESUMEN: La educación a distancia (EaD) fue reconocida por primera vez en la Ley de Directrices y Bases de la Educación (LBD) en 1996, y se refiere a una modalidad de enseñanza-aprendizaje en la cual los alumnos y sus tutores están separados y sin embargo conectados por medio de diversas tecnologías como Internet. La EaD ha

1Universidade Estadual de Campinas (Unicamp), Campinas - SP - Brasil. Professora Doutora da Área de Enfermagem na Saúde da Mulher e do Recém-nascido/FENF. Doutora pela Faculdade de Medicina de Ribeirão Preto da Universidade de São Paulo-USP. E-mail: eztanaka@unicamp.br

${ }^{2}$ Universidade Estadual Paulista (Unesp). Doutora em Fisioterapia. E-mail: dulcevb.sartori@gmail.com ${ }^{3}$ Universidade Estadual Paulista (Unesp), Botucatu - SP - Brasil. Mestre em Bases Gerais da Cirurgia pela Faculdade de Medicina de Botucatu. Coordenadora do curso de fisioterapia da Faculdade Anhanguera de Bauru. E-mail: larissa_rf_1903@hotmail.com

${ }^{4}$ Faculdade Anhanguera de Bauru, Bauru - SP - Brasil. Aluno especial de doutorado pelo programa Educação escolar da Faculdade de Ciências e Letras de Araraquara e aluno especial do programa Educação para Ciência da Faculdade de Ciências de Bauru. Mestre em Ciências na área de Microbiologia pela USP em 2012. É professor e coordenador do curso de Ciências Biológicas da Faculdade Anhanguera de Bauru. E-mail: lucasjbermejo@hotmail.com 
crecido gradualmente a lo largo de los años, y ha sido constante su uso en diferentes modalidades de cursos, tales como los técnicos, los de graduación o pos graduación. Los cursos de enfermería, así como otros cursos del área de la salud han utilizado la EaD con la finalidad de facilitar el acceso al aprendizaje, ofreciendo al alumno enseñanza de calidad y el conocimiento de nuevas tecnologías. El estudio fue desarrollado con el objetivo de analizar la EaD, así como su aplicación en los cursos de enfermería. Para ello se llevó a cabo una revisión literaria cualitativa de investigación teórica. Se analizaron artículos de bases de datos nacionales publicados en el periodo de 2002 a 2014.

PALABRAS CLAVE: Educación a distancia. Enfermeira. Aplicabilidad en enfermeira.

ABSTRACT: Distance education (EaD) was first recognized in the Law of Guidelines and Bases of Education (LBD) in 1996 and refers to a teaching-learning modality where students and tutors are separated yet connected through various technologies, like the internet. Distance Education has been growing steadily over the years, and its use has been constant in various modalities of courses, whether technical, undergraduate or postgraduate. Nursing courses, as well as other courses in the health area, have used it in order to facilitate access to learning, offering the student quality teaching and knowledge of new technologies. This study was developed with the purpose of analyzing Distance Education, as well as its application in the nursing courses. For that, a qualitative literature review was carried out. National databases published from 2002 to 2014 were withdrawn.

KEYWORDS: Distance education. Nursing. Applicability in nursing.

\section{Introdução}

A Educação a Distância $(\mathrm{EaD})$ refere-se a um processo de ensino-aprendizagem que se dá por meio tecnologias, estando os estudantes e tutores separados, de forma física, espacial e/ou temporal, contudo conectados através de tecnologias, a exemplo da Internet, ou mesmo utilizando outros meios, como: correio, rádio, televisão, vídeo, CD-ROM, entre outras tecnologias similares. Com o intuito de promover uma linguagem autônoma, a linguagem utilizada é diferenciada, procurando enfatizar processos pedagógicos interativos. Sendo assim, o papel dos tutores é de manter a motivação com estratégias de ensino com foco no aluno, o qual deve se identificar como principal responsável pelo aprendizado, buscando a criatividade e o pensamento reflexivo (MORAN, 2005; PALLOF, 2007).

Cursos técnicos, de graduação e pós-graduação, assim como outros cursos de formação profissional na modalidade $\mathrm{EaD}$ estão se firmando no Brasil com o intuito de atender a necessidade da universalização do acesso ao ensino de qualidade. O Ministério da 
Educação constituiu a Secretaria de Educação a Distância, voltada para a ampliação da oferta de ensino superior gratuito e de qualidade no país (BRASIL, 2005).

A enfermagem tem como principal atenção o cuidado com a saúde. O curso de graduação em enfermagem forma um profissional capaz de planejar, organizar, supervisionar e executar assistência de enfermagem ao cliente e à comunidade, preparando-o para atuar com eficiência em diferentes áreas de atenção à saúde e capacitando-o para intervir em problemas/situações de saúde fornecendo assistência necessária aos indivíduos e grupos de indivíduos no processo saúde e doença.

A enfermagem, como vários cursos de graduação, tem se apropriado da modalidade EaD. A Educação a distância tem permitido a capacitação dos profissionais de forma interativa, permitindo ao mesmo tempo o conhecimento de novas tecnologias (CAMACHO, 2009; ROJO et al 2011).

Camacho (2009) avaliou a "Educação a distância na Disciplina de Legislação, Ética e Exercício de Enfermagem", cujo objetivo foi descrever a inserção da disciplina de Legislação, Ética e Exercício de Enfermagem online no curso de graduação em Enfermagem da Universidade Estácio de Sá no estado do Rio de Janeiro, da qual participaram 45 alunos. Silva (2007) objetivou em seu estudo investigar a compatibilidade dos ambientes virtuais para o ensino-aprendizagem em enfermagem psiquiátrica, através do desenvolvimento e aplicação de curso online sobre transtornos de humor e personalidade, em uma amostra composta por 32 acadêmicos do curso de Bacharelado da Escola de Enfermagem de Ribeirão Preto - USP. Ambos os autores concluem que ambientes virtuais de aprendizagem são efetivos para apoiar o ensino das disciplinas estudadas em cada estudo.

Contudo, os estudos e publicações referentes ao tema proposto ainda são escassos na realidade dos cursos de enfermagem no Brasil.

Baseado nesses achados e considerações, este estudo foi desenvolvido objetivando analisar a educação a distância, assim como o uso dessa modalidade de ensino nos cursos de enfermagem no Brasil, verificando ao mesmo tempo em quais disciplinas nos cursos de enfermagem essa modalidade tem sido aplicada e qual o nível de conhecimento alcançado pelos alunos dos cursos de enfermagem. Para tanto, realizamos uma revisão de literatura de pesquisa teórica.

Tratou-se de uma Revisão não sistemática de literatura realizada nas bases de dados Scielo (nacionais), com recorte temporal de janeiro de 2002 a dezembro de 2015, com os argumentos de pesquisa: enfermagem a distância. A busca retornou 45 artigos, mas alguns 
fora do escopo da pesquisa. Foram excluídos resumos de pesquisas, teses, artigos de jornais e de revistas que não possuíam caráter científico.

Foi realizada a leitura do material consultado e em seguida o fichamento do conteúdo, selecionando então os pontos relevantes e o agrupamento para a inclusão na pesquisa.

\section{A educação a distância no Brasil}

Vários são os conceitos que a educação a distância recebeu ao longo dos anos, desde a sua consolidação, todos apresentando pontos em comum; contudo, cada um destacando pontos específicos de acordo com sua conceitualização, como a distância entre professor e aluno, a separação física entre professor e aluno, as formas de estudo na educação a distância (ALVES, 2011).

Desde o seu aparecimento e consolidação a partir do século XIX, a educação a distância vem crescendo substancialmente, começando por cursos oferecidos por correspondência; com os avanços tecnológicos e aparecimento do rádio e televisão, várias instituições passaram a oferecer alguma forma de ensino por meio destes (MORAN; 2008; ALVES, 2011).

A educação a distância ganhou uma nova perspectiva com o aparecimento das Tecnologias de Informação e Comunicação (TICs), facilitando o acesso ao ensino devido à sofisticação, rapidez e melhor aproximação entre professor/tutor e aluno (MUGNOL, 2009).

No Brasil, no ano de 1996 essa modalidade foi regulamentada por meio do Decreto de Lei 9.394 de 20 de dezembro do mesmo ano, em seu artigo 80, autorizando a Educação a Distância como modo de ensino. Em 2005, a educação a distância recebeu uma definição oficial por meio do Decreto 5.622, de 19 de dezembro (BRASIL,1996; BRASIL, 2005):

Artigo $1^{\circ}$ - Para os fins deste Decreto, caracteriza-se a educação à distância como modalidade educacional na qual a mediação didático-pedagógica nos processos de ensino e aprendizagem ocorre com a utilização de meios e tecnologias de informação e comunicação, com estudantes e professores desenvolvendo atividades educativas em lugares ou tempos diversos.

Em seu segundo parágrafo o mesmo artigo ressalta a obrigatoriedade de momentos presenciais nas situações específicas a seguir: avaliação dos estudantes; estágios obrigatórios, quando previstos na legislação pertinente; defesa de trabalhos de conclusão de curso, quando previstos na legislação pertinente; e atividades relacionadas a laboratórios de ensino, quando for o caso (BRASIL, 2005). 
As definições de quais níveis de educação poderão ofertar a educação vem logo no artigo segundo do mesmo Decreto 5.622, de 19 de dezembro de 2005 (BRASIL, 2005):

Artigo $2^{\circ}$ - A educação a distância poderá ser ofertada nos seguintes níveis e modalidades educacionais:

I - Educação básica, nos termos do art. 30 deste Decreto;

II - Educação de jovens e adultos, nos termos do art. 37 da Lei no 9.394, de 20 de dezembro de 1996; pertinentes;

III - educação especial, respeitadas as especificidades legais

IV - Educação profissional, abrangendo os seguintes cursos e programas:
a) sequenciais;
b) de graduação;
c) de especialização;
d) de mestrado; e
e) de doutorado.

a) técnicos, de nível médio;

b) tecnológicos, de nível superior;

V - Educação superior, abrangendo os seguintes cursos e programas:

A educação a distância tornou-se um meio promissor em meio à sociedade capitalista. A modalidade que antes era oferecida em instituições públicas como forma de capacitação de professores da rede pública passou ser vista pelas instituições privadas como um bom investimento de mercado. Cursos presenciais que tinham apenas parte da sua carga horária oferecida a distância, passaram a ser oferecidos em sua totalidade a distância, sendo os primeiros os cursos de pedagogia e normal superior, como administração. Atualmente, a educação a distância tem penetrado em vários contextos educacionais (GIOLO, 2008; LAPA; BELLONI, 2012).

A educação a distância tem sido uma excelente alternativa para a progressão na carreira, proporcionando oportunidade de ensino, seja ele em nível médio, técnico, superior ou mesmo complementar. Essa modalidade proporciona flexibilidade de tempo e espaço, não exigindo a presença em local e horários em que a aula foi ministrada, podendo a mesma ser acessada por meio de um computador, facilitando assim o acesso à Educação (MORAM, 2009; MUGNOL, 2009).

Existe ainda certo preconceito em relação ao uso da educação a distância, contudo é possível afirmar que as novas tecnologias de informação e comunicação (TICs) tem modificado o paradigma do ensino/aprendizagem e das relações entre o indivíduo, o trabalho e a sociedade. Assim, a EaD tem se tornado uma ferramenta adequada de ensino para atender as demandas de formação e atualização profissionais hoje existentes (MARTINS et al, 2010). 


\section{A educação a distância nos cursos de enfermagem no Brasil}

No Brasil, a escola de enfermagem já conta com diversas experiências envolvendo de alguma forma a modalidade de educação a distância, no desenvolvimento de softwares para estudantes de graduação, com o intuito de capacitá-los a desenvolver atividades práticas, como também tem demonstrado um crescimento na produção científica nessa área de forma constante, havendo uma tendência ao desenvolvimento de ambientes virtuais de aprendizagem (AVAs) a partir de pesquisas de mestrado e doutorado, predominando a capacitação do enfermeiro na área assistencial (RODRIGUES \& PERES, 2008; MASTINS et al, 2010).

Rodrigues e Peres (2013) realizaram uma pesquisa metodológica de desenvolvimento tecnológico com o intuito de desenvolver um ambiente virtual de aprendizagem (AVA) para a educação continuada em enfermagem sobre ressuscitação cardiorrespiratória em neonatologia. O ambiente foi avaliado por estudantes de graduação em enfermagem, profissionais da área da tecnologia da informação, assim como especialistas em educação, neonatologia e terapia intensiva, recebendo avaliação positiva e mostrando-se útil para o auxílio da aprendizagem, desenvolvimento de novas habilidades e capacitação do profissional de enfermagem.

No ano de 2003, Cassiani e colaboradores descreveram o desenvolvimento de um curso on-line sobre o processo de administração de medicamentos. O curso recebeu avaliação positiva dos estudantes de graduação da Escola de Enfermagem da USP de Ribeirão Preto, recebendo comentários como "acho que é uma ótima forma de aprendizado e facilita o acesso ao material pelo aluno" e "Muito interessante esse curso via Internet, principalmente por abordar o tema de forma clara. Assim podendo ser usado por alunos em fase de aprendizado e também por profissionais em caso de dúvida".

Cogo et al (2009), em um estudo transversal exploratório que buscou investigar de forma sistematizada a opinião de docentes sobre objetos educacionais digitais em oxigenoterapia na área de enfermagem, verificaram que os docentes foram favoráveis à apresentação desses objetos. Os autores afirmam ainda que a implementação dessas novas tecnologias exige dos docentes disposição em aperfeiçoar seus métodos de ensino, baseados em ações e desafios frente a uma nova maneira de ensinar, assim como permite ao aluno o despertar de novas habilidades como a criatividade, a criticidade e autonomia de pensamentos. Assim, para eles, os objetos educacionais digitais promovem ousadia na busca de novos conhecimentos que capacitem os alunos de enfermagem a avançar na construção de sua própria aprendizagem. 
Bastos e Guimarães (2003) relatam a experiência como tutoras de uma disciplina do curso de mestrado "Maestría em Administración de Servicios de Enfermería", da Universidad Nacional de Rosario, Argentina/Facultad de Ciencias Médicas/Escuela de Graduados, na modalidade a distância. As autoras levantam pontos importantes percebidos em sua experiência como tutoras de uma disciplina na modalidade a distância numa pós-graduação em enfermagem. Um dos pontos observados foi a necessidade de dispor aos alunos recursos instrucionais mais flexíveis que permitissem aos mesmos um aprendizado independente e autônomo.

Basto e Guimarães apontam também as diferenças entre o contato com os alunos: diferente da educação presencial que o contato com os alunos é coletivo, na educação a distância esse contato é individualizado, tutor/aluno, evidenciando a necessidade de o tutor saber trabalhar com as diferenças do ponto de vista profissional, cultural e de habilidades.

Baseadas nessa experiência, as autoras concluem o artigo com uma visão positiva do uso da educação a distância na enfermagem:

Com o olhar focado na realidade da Enfermagem, as tutoras acreditam que a EAD pode se constituir em ferramenta pedagógica adequada para qualificar enfermeiros que, por razões várias, não têm acesso aos processos convencionais de pós-graduação. Ela viabiliza a qualificação de um grande contingente de enfermeiros, que estão geograficamente dispersos, longe dos grandes centros, sem possibilidade de afastar-se do seu cotidiano de vida e profissional. Frente à necessidade de formar os atendentes de enfermagem do país, que é o desafio de todos nós, e de qualificar enfermeiros para atuar como docentes, a EAD apresenta-se como uma ferramenta pedagógica eficaz e possível (BASTOS; GUIMARÃES, 2003, p.690).

$\mathrm{Na}$ enfermagem, assim como em qualquer outro curso que ofereça a modalidade a distância, o tutor torna-se importantíssimo: ele tem o papel de mediador, interpretando o conteúdo junto ao aluno, estimulando o mesmo a prosseguir, esclarecendo as dúvidas, participando ao mesmo tempo do processo de avaliação da aprendizagem. É ele quem garante a inter-relação do aluno no sistema, viabilizando a articulação necessária entre os elementos do processo e a execução dos objetivos propostos (PRADO et al, 2012).

É observado um progresso da escola de Enfermagem no Brasil na modalidade de educação a distância, no que se refere a materiais em AVAs em cursos de capacitação e disciplinas ofertadas na graduação. Contudo, levando em consideração a pequena quantidade de publicações existente, podemos considerar que o crescimento dessa modalidade na enfermagem ainda é tímido (ROJO et al, 2011) 
No ano de 2008, Rodrigues e Peres identificaram em um estudo exploratório apenas dois cursos oferecidos por IES cadastrados no Ministério da Educação (MEC), sendo eles dois cursos de pós-graduação latu-sensu. Um outro estudo realizado por Rojo e colaboradores em 2011, sobre o panorama da educação a distância em enfermagem no Brasil, nos mesmos moldes do primeiro, verificou a existência de dois cursos de graduação em enfermagem e nove de pós-graduação oferecidos na modalidade a distância.

Análises do panorama da Educação a Distância no Brasil em enfermagem mostram um crescimento ainda pequeno dessa modalidade no Brasil, levantando a necessidade de investimento e capacitação tecnológica dos docentes e discentes por parte das Instituições de Ensino Superior (IES), assim como a implementação de infraestrutura necessária ao desenvolvimento (CAMACHO, 2009).

A educação a distância torna-se uma excelente alternativa para a capacitação dos profissionais de enfermagem, que em sua maioria trabalham em turnos de 12 horas, e realizam mais de uma atividade empregatícia, não dispondo de tempo para estar em uma sala de aula em local e horário específico, como necessário na educação presencial.

\section{Considerações finais}

É possível verificar que educação a distância é uma modalidade em constante expansão no mundo todo, sendo uma alternativa que oferece educação de qualidade com flexibilidade de tempo e espaço. O surgimento de novas tecnologias de informação e comunicação contribuem, facilitando ainda mais o desenvolvimento EaD de ensino. Contudo, é necessário a preparação das instituições de ensino frente a essa modalidade inovadora, capacitando os professores e alunos a desenvolver uma maneira de aprendizagem adequada.

A educação a distância tem estado presente em vários níveis de educação, contudo prevalece na educação superior, em cursos de graduação, pós-graduação, capacitação de profissionais e educação continuada. No curso de enfermagem, essa modalidade tem avançado com o passar dos anos. A modalidade é utilizada na aplicação de disciplinas nos cursos de graduação em enfermagem presencial, como forma complementar, em cursos de pós-graduação, latu sensu e stricto sensu. Contudo, ainda demonstra um crescimento relativamente pequeno.

Por se tratar de uma profissão que envolve situações práticas, talvez haja ainda um certo receio em implantar a educação a distância como modalidade de ensino. Contudo, estudos têm demonstrado a boa aceitação de disciplinas e cursos em enfermagem por meio da 
modalidade a distância, sendo essa uma maneira de melhor capacitar o profissional de enfermagem, que tanto necessita manter-se atualizado frente às inovações que surgem frequentemente na área da saúde.

São esperadas mudanças no que se refere à educação a distância em enfermagem no Brasil, devido aos grandes avanços tecnológicos e à necessidade de capacitação do profissional de enfermagem, sendo a educação a distância uma nova estratégia de ensino e capacitação do profissional, que recebe do competitivo mercado de trabalho atual a cobrança por profissionais cada vez mais capacitados, com domínio no seu campo de atuação.

\section{REFERÊNCIAS}

ALVES, L. Educação à Distância: Conceitos e história no Brasil e no mundo. RBAAD Associação Brasileira de Educação à Distância. v. 10, 2011.

BASTOS, M.A.R; GUIMARÃES, E.M.P. Educação a distância na área da enfermagem: relato de uma experiência. Revista Latino-am Enfermagem, v. 11, n. 5, 2003.

BRASIL. Ministério da Educação. Disponível em: <http://portal.mec.gov.br/>. 2011. Acesso em: 21 fev. 2015.

BRASIL. Ministério da Educação. Curso piloto. Disponível em:

<http://www.uab.mec.gov.br/cursopiloto.php>. Acesso em: 26 fev. 2015.

BRASIL. Decreto 5.622, de 19 de dezembro de 2005. Regulamenta o artigo 80 da Lei no 9.394, de 20 de dezembro de 1996, que estabelece as diretrizes e bases da educação nacional. Diário Oficial [da] República Federativa do Brasil. Brasília, DF, 20 dez. 2005. Disponível em: <http://www.planalᄀto.gov.br/ccivil_03/_Ato20042006/2005/Decreto/ D5622.htm>. Acesso em: 26 fev. 2015.

BRASIL. Lei $\mathrm{n}^{\circ}$ 9.394, de 20 de dezembro de 1996. Estabelece as diretrizes e bases da educação nacional. Presidência da República da Casa Civil. Brasília, 20 de dez 1996. Disponível em :< http://www.planalto.gov.br/ccivil_03/leis/19394.htm>. Acesso em: 26 fev. 2015.

CAMACHO, A. C. L. F. Educação a distância na Disciplina de Legislação, Ética e Exercício de Enfermagem. Revista Brasileira de Enfermagem, v. 62, n.1, Brasília, 2009.

CAMACHO, A. C. L. F. Análise das publicações nacionais sobre educação à distância na enfermagem. Revista Brasileira de Enfermagem, v. 62, n. 4, Brasília, 2009.

CASSIANI, S.H.B. et al. Desenvolvimento de um curso on-line sobre o tema administração de medicamentos. Rev. Esc. Enferm USP, v. 37, n.3, 2003. 
COGO, A. L. P. Objetos educacionais digitais em enfermagem: Avaliação por docentes de um curso de graduação. Rev. Esc. Enferm USP, v.43, n. 2. 2009.

GIOLO, J. A educação a distância e a formação de professores. Edu. Soc. Campinas, v.29, n. 105, p. 1211-1234, set/dez., 2008.

LAPA, A. B.; BELLONI, M. L. Educação à distância como mídia educação.

Perspectiva, v. 30, n.1, 2012.

MARTINS, T. Y. C.; RIBEIRO, R. C.; PRADO, C. Transdisciplinaridade na educação à distância: Um novo paradigma no ensino da enfermagem. Revista Brasileira de Enfermagem, v. 64, n. 4. Brasília, 2011

MORAN, J. M. O que é educação a distância? Disponível em:

<http://umbu.ied.dcc.ufmg.br/moodle/file.php/117/Nivel_0/Conteudo/O_que_ededuc_a _distancia.pdf>. Acesso em: 21 fev. 2015.

MORAM, J. M. Modelos e avaliação do ensino superior à distância no Brasil. ETD Educação Temática Digital, v.10, n.2, p.54-70, jun. 2009.

MUGNOL, M. A educação a distância no brasil: conceitos e fundamentos. Rev. Diálogo Educ., v. 9, n. 27, 2009.

PALLOF, R. M.; PRATT, K. O aluno virtual: um guia para trabalhar on-line. Porto Alegre: Artmed; 2004.

PRADO, C. et al, 2012. Espaço virtual de um grupo de pesquisa: o olhar dos tutores. Rev Esc Enferm USP, v.46, n.1, 2012.

RODRIGUES, R. C. V.; PERES, H. H. C. Desenvolvimento de Ambiente Virtual de Aprendizagem em enfermagem sobre ressuscitação cardiorrespiratória em neonatologia. Rev Esc Enferm USP, v.47, n.1, 2013.

RODRIGUES, R. C. V; PERES, H. H. C. Panorama brasileiro do ensino de Enfermagem On-line. Rev Esc Enferm USP, v. 42, n.2, 2008.

ROJO, T. R. et al. Panorama da educação à distância em enfermagem no Brasil. Rev Esc Enferm USP, v. 43, n. 6. Riberirão Preto, 2011.

SILVA, E. C. Educação à distância: Ambientes digitais para o processo ensinoaprendizagem em enfermagem psiquiátrica. 2007. Tese (Doutorado em Enfermagem). Escola de Enfermagem de Ribeirão Preto. Universidade de São Paulo, Ribeirão Preto. 


\section{Como referenciar este artigo}

ZAMBRANO TANAKA, Erika et al. A educação a distância nos cursos de graduação em enfermagem: aplicação e efetividade. Revista on line de Política e Gestão Educacional, Araraquara, v. 21, n. esp. 1, p. 831-841, out./2017. Disponível em: <http://dx.doi.org/10.22633/rpge.v21.n.esp1.out.2017.10455>. ISSN: 1519-9029.

Submetido em: 10/05/2017

Aprovado em: 15/09/2017 